\title{
TENDENCIAS MIGRATORIAS INTERNACIONALES DE HONDURAS
}

Manuel Antonio Flores Fonseca. Universidad Nacional Autónoma de Honduras, Facultad de Ciencias Económicas, Instituto de Investigaciones Económicas y Sociales (UNAH-FCE-IIES), Ciudad Universitaria, Edifício C2, Primer piso, Tel/Fax: 504-2239 1849

E-mail: mflores@iies-unah.org

\section{RESUMEN}

La migración siempre ha estado presente en la sociedad hondureña, teniendo cambios en sus tendencias, que pueden delimitarse históricamente desde la época del descubrimiento y conquista, pasando por la época republicana hasta la finalización del enclave minero y bananero como de inmigración, para después convertirse en emigración partiendo de la búsqueda de oportunidades primeramente de la población garífuna y mestiza conectada de la economía de enclave, principalmente a los puertos de la costa este de los Estados Unidos de América. Con el tiempo esta emigración llevó incluso a alcanzar en los años sesenta por la población salvadoreña en la búsqueda de tierras y trabajo y en los años setenta y ochenta por los conflictos armados de El Guatemala, El Salvador y Nicaragua, más que todo como refugio. Finalmente la tendencia migratoria actual hondureña es la emigración, misma que se genera en los años noventa con la implementación de las políticas neoliberales que deprimen algunos sectores económicos, entre ellos el agrícola y generan una gran masa de jóvenes sin empleo o cuentapropistas muy proclives a la búsqueda de oportunidades en las regiones más desarrolladas del país y en gran medida en el exterior, principalmente en los Estados Unidos de América, a esto se suma el azote de los desastres naturales (Mitch) que como evento histórico visibiliza la emigración y a partir de ese momento se entroniza en la población hondureña alcanzado dimensiones de importancia, ya diversifica sus destinos y produce un flujo de remesas que introduce al país en el top mundial de proporciones más altas con respecto al Producto Interno Bruto.

Palabras clave: Migración Internacional, Demografía, Tendencias migratorias. 


\title{
HONDURAS INTERNATIONAL MIGRATION TRENDS
}

\author{
Manuel Antonio Flores Fonseca. Universidad Nacional Autónoma de Honduras, \\ Facultad de Ciencias Económicas, \\ Instituto de Investigaciones Económicas y Sociales (UNAH-FCE-IIES), \\ Ciudad Universitaria, Edifício C2, Primer piso, Tel/Fax: 504-2239 1849 \\ E-mail: mflores@iies-unah.org
}

\begin{abstract}
Migration has been always present in Honduran society, whilst having changes in its trends, which can be historically delimited since the age of discovery and conquest, passing through the times of the Republic and into the mining and banana enclave as immigration, and later became emigration starting from the search of opportunities by Garifuna and Mestizo population connected to the enclave economy, headed to the east coast ports of the United States of America. In time, migration even became the highest in the region, while the country started transforming into an attractive place to immigrate in the seventies to the people of El Salvador who searched lands and jobs, and equally during the seventies and eighties due to the armed conflicts between Guatemala, El Salvador, and Nicaragua, as a refugee. Finally, the current migratory trend in Honduras is emigration, which was generated during the nineties with the introduction of neoliberal politics which depressed several economic sectors, such as agriculture, and generate a large mass of young people without employment or freelancers very inclined to find new opportunities in more developed areas of the country yet mostly overseas, like in the United States of America. Moreover the devastation caused by natural disasters (Hurricane Mitch) further caused that from that moment emigration was entrenched in Honduran population reaching important dimensions, destinations are diversified and a wave of remittance is produced, introducing the country into the global ranking of higher proportions with respect to the Gross National Product.
\end{abstract}

E\&A IIES

Keywords: International Migration, Demography, Migration trends. 


\section{INTRODUCCIÓN}

Las tendencias migratorias internacionales tienen comportamientos cambiantes en el tiempo, generados por factores económicos, sociales, políticos, culturales e incluso naturales que llevan a las poblaciones a cruzar las fronteras nacionales para buscar oportunidades económicas, educativas, sociales y de seguridad que lleven a mejorar la calidad de vida. Tomando como referencia a la migración internacional llegamos a preguntarnos cuales con esos factores, hechos, causas que han incidido en la población hondureña para ser parte activa de la migración y cómo han evolucionado a través del tiempo, tomando en cuenta no solamente los acontecimientos nacionales de diversa índole, sino acontecimientos o cambios que se producen con las otras naciones del mundo.

Desde nuestra perspectiva históricamente delimitamos los cambios en las tendencias de la migración así; desde el descubrimiento, conquista y colonia, la época republicana hasta la finalización del enclave minero y bananero como de inmigración, para después convertirse en una tendencia emigración principalmente hacia los Estados Unidos de América. Con el tiempo esta emigración fue relevante, la población salvadoreña en la búsqueda de tierras y trabajo y en los años setenta y ochenta por los nacionales de los países vecinos por los conflictos armados de Centroamérica.

Finalmente, la tendencia migratoria actual es la emigración, misma que se genera en los años noventa con la implementación de las políticas neoliberales, que deprimen algunos sectores económicos, entre ellos el agrícola y generan una gran masa de jóvenes sin empleo o que trabajan por cuenta propia muy proclives a la búsqueda de oportunidades en el país o en el exterior, principalmente en los Estados Unidos de América, a esto se suma los desastres naturales, entre ellos el huracán Mitch, que en 1998 como evento histórico visibiliza la emigración y a partir de ese momento se entroniza en la población hondureña como estrategia de vida, que incluso ya diversifica sus destinos migratorios tradicionales.

Este ensayo quiere contribuir de manera exploratoria a generar conocimiento sobre la temática migratoria internacional y su relación acontecimientos históricos, sociales, económicos, políticos y de otra índole que contribuyen a producir tendencias migratorias a través del tiempo que culminan en el momento actual y que inciden en acontecer nacional. 


\section{MARCO DE REFERENCIA}

La migración como variable demográfica ha tenido una posición secundaria en los estudios de población, por su imposibilidad de incluirla en las relaciones analíticas y teóricas, las dificultades de medición e incluso la despreocupación de algunas escuelas de pensamiento demográfico (Welti, 1997, p. 123).

Cada vez más las migraciones adquieren relevancia y significación, son una constante en los medios de comunicación, producen una gran cantidad de publicaciones de diversas disciplinas, incluso ha aumentado las teorías explicativas. Estas teorías se inician en la Neoclásica, la nueva economía de las migraciones laborales, de los mercados duales, del sistema mundial, las redes migratorias, análisis de sistemas aplicados a las migraciones y la teoría de la causación acumulativa (Arango, 2003).

Tiene evidencia la migración desde el momento mismo de la aparición del hombre en la tierra que permitió poblar el mundo en sus continentes. Movimientos migratorios como el cruce del África a los otros continentes, el dominio ibérico con los movimientos europeos a América, el traslado forzoso de africanos a América, los desplazamientos de inicios del pasado siglo hacia América del Norte y Sur, y más reciente los movimientos hacia el norte del hemisferio desde el sur, como del oriente hacia los demás continentes, entre otros son desplazamientos de importancia.

En el año 2010 se estima en el mundo que hay aproximadamente 214 millones de migrantes internacionales, pero el porcentaje de migración internacional se ha mantenido estable $(3 \%$ de la población mundial) en los últimos 50 años. A su vez se calcula que 50 millones de personas_viven y trabajan en el extranjero de manera irregular y cada año 5 millones cruza fronteras internacionales. El grueso de los migrantes internacionales se produce entre países con niveles de desarrollo muy diferentes. El 37\% de la migración mundial desde países en desarrollo a países desarrollados. La mayoría del desplazamiento tiene lugar entre países de la misma categoría de desarrollo: alrededor del $60 \%$ de los migrantes se traslada o bien entre países en desarrollo o entre países desarrollados (el restante 3\% se mueve desde países desarrollados a países en desarrollo (PNUD, 2009, p.23). 
En Honduras, hay varias estimaciones de migrantes internacionales, en el caso de la inmigración no debería ser una complejidad actualmente, ya que existen registros de migración, aunque son de difícil acceso público, sin embargo, los censos de población del país han recogido información sobre el país de nacimiento y país donde residía cinco años antes del censo, en el caso del censo de población de Honduras del 2001 los nacidos en el exterior ascienden a 27,976 personas. No pasa lo mismo con las estimaciones de emigrantes, que son más complejas, las estimaciones van desde utilizar los censos y encuestas de los países de origen y de destino y la utilización de procedimientos indirectos demográficos. Las estimaciones conservadoras más recientes muestran una ancha franja de diferencias, el Banco Mundial 2010 los emigrantes 569,700, EPHPM 2010 estima 238,669, ACS 2009 625,000 de origen hondureño en EUA, Censo de población de EUA 2010 estima 633,401 de origen hondureño, censos de población de ronda 2010 se calcula 677,950 nacidos y de origen hondureño.

\section{FUENTES DE INFORMACIÓN}

Para el desarrollo de este ensayo se ha hecho un recuento de las principales fuentes de información

Dentro de las fuentes demográficas reciben especial mención los censos y recuentos de población del país, algunas encuestas de hogares nacionales que contienen módulos o información de migración y los censos de población, encuestas especiales y registros poblacionales de países de destino de la emigración hondureña. 


\section{RESULTADOS}

Para conocer las tendencias migratorias internacionales de Honduras hemos delimitado los principales periodos históricos, sociales y económicos que han influenciado algunos acontecimientos de carácter migratorio, esta clasificación propuesta se delimita en varios periodos así: descubrimiento, conquista y colonización, albores de independencia e inicios de la época republicana, reforma liberal y las economías de enclave, fin de la economía de enclave y entronización de dictaduras, creación del Estado moderno hasta guerra Honduras-El Salvador, conflictos armados en la región Centroamericana, liberalización de la economía, fenómenos naturales y tendencias recientes.

\subsection{Descubrimiento, conquista y colonización.}

Al momento de la conquista española, lo que actualmente es el territorio hondureño estaba habitado por grupos indígenas de dos tipos culturales: cacicazgos en el centro occidente y tribus en el oriente. Al momento del descubrimiento la población total sostenida por las diferentes economías indígenas pudo haber alcanzado una cifra de 1,396,858 personas, 851,260 en el occidente y centro del país, 528,970 en el oriente y 16,628 en otras áreas. Sin embargo, producto de la conquista, colonización y enfermedades se produjo un declive catastrófico en la población indígena que significó que al final del período colonial se estimaran solamente 60,000 personas (Newson, 1992).

Honduras, aunque es descubierta por Cristóbal Colón en 1502, pasa varias décadas hasta que el territorio fuera puesto bajo efectiva administración española, porque la conquista y colonización fue pospuesta hasta 1522 para tomar posesión del área. La población en los pueblos de españoles era reducida.

El descubrimiento de muchas vetas de metales a finales del siglo XVI y principios del XVII, produce una atracción de los españoles hacia esos lugares en los cuales forman pueblos, que también trae aparejado el traslado forzoso de esclavos negros de las islas del Caribe cuya procedencia real era África, que son traídos para trabajar en la extracción de minerales. 
Como los españoles deseaban conservar los derechos sobre el nuevo mundo a lo largo del periodo colonial emitieron decretos prohibiendo que extranjeros se asentaran o comerciaran en el nuevo mundo, sin embargo, los extranjeros aparecieron en Honduras, primero como agresores, luego como colonos y comerciantes ilegales.

Desde mediados del siglo XVI los barcos y puertos españoles, especialmente Trujillo y Puerto Caballos, fueron atacados por corsarios extranjeros, franceses e ingleses. A raíz de la guerra España e Inglaterra (1625) se incrementan la piratería e incluso llegaron a asentarse en Roatán y colonias cercanas al cabo de Gracias a Dios. En el siglo XVIII los ingleses consolidan la "Costa de los Mosquitos" convirtiéndola en Protectorado y aumenta la población no indígena, siendo sus primeros asentamientos en el río Tinto, Cabo de Gracias a Dios, Bluefields y Punta Gorda. En 1737 más de tres cuartas partes de los 1,123 habitantes de la costa vivían en el sector. En la zona se explotaba el comercio de la madera de tinte, caoba, zarzaparrilla y concha nacar y se exportaba a Inglaterra, Holanda y América del Norte. En 1757 se estima en la Costa de los Mosquitos 154 blancos, 170 mulatos y mestizos, 800 esclavos negros, para un total de 1,124 habitantes. A partir de la ofensiva española por retomar el territorio logran recuperar Roatán y llegar al rio Tinto y los ingleses entre 1783-1786 empiezan a evacuar la costa, aunque mantuvieron contactos comerciales de contrabando con los zambos-mosquitos desde Jamaica y Belice.

Un dato relevante es que entre 1787 hasta 1800 los españoles intentaron controlar la costa de los mosquitos estableciendo colonias de inmigrantes españoles en rio Tinto, Cabo de Gracias a Dios y Bluefields que reclutados en España y las Islas Canarias trataron de poblar la zona, pero resultó infructuoso su intento. Otros inmigrantes negros habían llegado a la zona en 1776 cuando negros franceses recibieron asilo y se asentaron en Trujillo y en 1797 se unen 1,490 negros Caribe de St. Vincent, a estos grupos se le unieron los esclavos negros que permanecieron en la zona (Newson, 1992). 


\subsection{Albores de independencia e inicios de la época republicana.}

La recolección de información demográfica había comenzado con los registros surgidos en la época de la Colonia, cuando los intereses de los conquistadores y los registros para fines tributarios, se convierten en las primeras fuentes de información. Luego es la iglesia católica la que realiza las labores de recolección de información de los poblados en su jurisdicción. El considerado primer recuento censal es levantado por el Obispo de Comayagua en 1791, donde la población no superaba los cien mil habitantes. También en los censos de población del siglo XIX, miembros de la iglesia participan activamente en su elaboración.

La independencia de Honduras de España se declara en 1821, aunque en años cercanos ingleses se establecen en las orillas de Rio Tinto y las autoridades de Trujillo no hicieran nada para oponerse. En el período post independentista, se realizaron algunos censos y recuentos estadísticos de las personas, sin embargo, en ese período de reorganización gubernamental se perciben claras deficiencias en calidad y en la periodicidad de la recolección censal.

La más antigua de las legislaciones de la inmigración alude a José Cecilio del Valle en 1825 en la denominada "Ley de Colonización" emitida al amparo de la Constitución Federal de 1824. Bajo esta legislación empiezan a llegar los primeros inmigrantes principalmente a Tegucigalpa, Comayagua, Puerto de Amapala. El explorador Wells encontró en 1857 en Amapala a italianos, alemanes y estadounidenses trabajando o haciendo negocios, aunque su presencia era pequeña. En 1866 el presidente Medina emitió una nueva Ley de Inmigración con privilegios y facilidades para extranjeros (Amaya, 2000).

Después de dos cuartos de siglo de vida independiente, el país había entrado en una etapa de estancamiento, ya que no se había organizado políticamente y gubernamentalmente, por lo que adolecía de una infraestructura para insertarse económicamente en el plano mundial, esto lleva a las administraciones de Soto y Bográn (1876-1891) a iniciar una reforma liberal de transformación del país para pasar de una economía de subsistencia a una economía incipientemente capitalista, que incluía también favorecer la inmigración. 


\subsection{De la reforma liberal y las economías de enclave.}

La reforma liberal también es una etapa de mejoramiento en la recolección de información demográfica, no sólo por la creación de una institución generadora de Estadísticas, sino también por la producción de censos y estadísticas de mejor calidad (1887-1895), entre ellos la realización del primer anuario estadístico de Honduras en 1889 y la inclusión de información sobre el número de extranjeros en los censos de población de 1887 y 1895 , que asciendan a 6,167 y 6,221 respectivamente, principalmente centroamericanos y europeos. (Flores, 1990).

Las administraciones de la reforma liberal querían orientar la economía a la expansión minera, por lo que se hicieron los arreglos legales para hacer atractiva la llegada de compañías extranjeras y se le otorgan una serie de facilidades que van desde exoneraciones, exenciones por concepto de exportaciones e importaciones y concesiones. Así se inicia el segundo auge minero, pero esta vez con tecnología más avanzada, con grandes recursos económicos, técnicos y empresariales, lo que hace aparecer el capital extranjero, entre ellos el norteamericano y en menor medida el capital inglés y francés. Este predominio de la inversión extranjera en forma de enclave minero comprende desde mediados de la década de 1880 hasta fines de ese siglo (Murga, 1978).

La administración de Soto recibe la colaboración de extranjeros para que ayuden en el desarrollo técnico y administrativo, entre ellos habían cubanos y de los países centroamericanos. También en la reforma liberal llegan españoles, militares que organizan la escuela militar, profesores que renuevan la universidad, escuelas normales de varones y de señoritas, la de artes y oficios, la tipografía nacional. Asimismo, algunos españoles hacen florecer el cultivo del tabaco en el occidente del país.

Después de las administraciones de Soto y Bográn que fueron proclives a la inmigración, sus sucesores Ponciano Leiva y Policarpo Bonilla alentaron a la inmigración, principalmente el último de ellos decreta una nueva Ley de Inmigración en 1895, que fue la que empieza a observar limitados flujos inmigrantes, pero no es sino hasta la Ley de Inmigración de 1906 que facilita la llegada de extranjeros, preferentemente europeos y estadounidenses, aunque no impedían extranjeros de otras latitudes. Debido a que inmigrantes árabes fueron controlando el comercio del país, se crea suspicacias entre 
los nacionales, por esta razón se promulga una nueva Ley de Inmigración en 1929, con elementos discriminatorios para ciertas nacionalidades, como árabes, chinos y negros. Los judíos llegan en mayor medida a partir de los años treinta a Honduras (Amaya, 2000).

En los últimos años del Siglo XIX e inicios del Siglo XX se produce una contracción del mercado de la plata, que produce el deterioro del auge minero, haciendo que el capitán extranjero busque un nuevo rumbo, es así que empieza el auge de la agricultura, especialmente el banano en el litoral atlántico del país, que ya había empezado a desarrollarse por pequeños cultivadores y algunos inmigrantes.

Hasta el año 1866 el banano era desconocido en los mercados de Estados Unidos y en ese año se empezaron a importar bananos a Nueva York y poco a poco fue ganando consumidores norteamericanos. La demanda por ese fruto se extiende de la zona del Caribe hacia Centroamérica y es allí donde empieza el auge de su cultivo en Honduras, primeramente en Islas de la Bahía y después la zona continental.

La historia del cultivo del banano se inicia con pequeños productores, sin embargo, sobresale la historia de la grandes corporaciones norteamericanas productoras de la fruta, siendo esta penetración en agricultura producto de limitaciones del régimen de producción de los pequeños productores, ya que la demanda aumentaba y el mercado se extendió y el escenario de la comercialización fue copado por grandes empresas con innovaciones administrativas, técnicas, de transporte y cuidado de la fruta y finalmente la introducción de las empresas en la producción, entre 1899 y 1905, mediante beneficios que reciben a través de concesiones y se convierten en una fuerza productiva superior a los productores locales y comienzan a relacionarse con la política local y sus posibilidades de inversión en gran escala, tendencia que lleva a un mayor control de concesiones de tarea y sobre la producción agrícola que se entroniza con mayor vigor en la primera década del siglo y se van atando con las concesiones ferroviarias.

Entre el año 1896-1903 las exportaciones de bananos del total se duplicaron, lo mismo que el comercio entre los puertos hondureños y los norteamericanos, llegando en 1920 a triplicar las exportaciones de esta fruta con respecto a los demás países productores. Llegó a convertirse en la "primera economía 
de bananos del mundo" y las exportaciones de bananos opacaron a la de los minerales. La expansión norteamericana en Honduras no solo incluyó a las actividades agrícolas y las conexas del ferrocarril, sino que se ensanchó como enclave a otras actividades, entre estas la producción de cerveza, grasas vegetales, calzado, etc.

En este mismo periodo de tiempo empiezan a llegar al país inmigrantes de Europa, entre ellos alemanes, ingleses, judíos y del medio oriente, entre ellos palestinos, árabes y turcos que se incorporan en algunas actividades productivas y en el comercio urbano y van formando actividades un poder económico secundario, los alemanes en el sur y los árabes y judíos en el norte del país. Poco a poco van controlando una parte del comercio de exportación, parte del de importación y la totalidad del comercio interior al menudeo.

Beneficiados por las conexiones y conocimientos comerciales, la movilidad geográfica, así como por la iniciativa que tenían aprovechan las coyunturas económicas comerciales favorables de 1880 a 1895 en el sur y de 1910 en el norte, los inmigrantes expanden su base económica. Desde esa época poco a poco su inserción en el proceso productivo se va haciendo más notoria y podría decirse que adquieren poder económico a través de introducirse en varias ramas económicas, esto quedó más evidenciado (porque en el sur el capital alemán fue confiscado en la segunda guerra mundial), quedando solamente en libertad el capital de las otras nacionalidades, entre ellos árabes y judíos, principalmente en el norte del país, principalmente en San Pedro Sula, donde los inmigrantes han contribuido a la expansión de la actividades productivas y comerciales.

Los inicios del siglo XX no significaron grandes mejorías en la captación de estadísticas demográficas, sino más bien se dio paso a la realización de recuentos censales de dudosa aseveración. Se realizaron censos de población en los años 1901, 1905, 1910, 1916 que no recogieron información sobre extranjeros en el país.

Un grupo de inmigrantes búlgaros se establecieron en el país en las primeras décadas del siglo $\mathrm{XX}$, estos como otras nacionalidades llegaron al país en el periodo de expansión bananera, tal es el caso que entre 1903 a 1920 los Italianos Vaccaro y DÁntoni fundaron la compañía Standard Fruit 
Company con sede en La Ceiba. Esta ciudad necesitaba de muchos nuevos trabajadores y comienza a crecer económicamente. La producción bananera creció y para traerla al puerto tuvieron que construir un ferrocarril, lo que incidió para que se trajeran trabajadores de Italia, pero también en pequeñas cantidades de los Balcanes, entre ellos los búlgaros. Estos incursionan en la zona montañosa adyacente a la ciudad, llena de cascadas y rodeadas de selva, que en esa época estaba sin explorar, llegando a nombrar a un rio desconocido con el nombre de río Bulgaria, único hecho en el continente americano, donde los inmigrantes búlgaros influyeron a su toponimia (Corrales, 2010). Los censos hondureños que recogen información de esta migración, en 1930 ascienden a 53 búlgaros, en los siguientes censos de 1935, 1940 y 1945 su presencia asciende a 28 en promedio en los tres censos, ubicándose geográficamente básicamente en los departamentos de Atlántida y Yoro.

\subsection{Fin de la economía de enclave y entronización de dictadura.}

Al mismo tiempo muchos años de guerras intestinas en la disputa del poder, desembocan en la llegada al poder del caudillo Carías que se perpetua en el poder varios años (1933-1948), en medio de la depresión mundial, fortalece las Fuerzas Armadas, mantiene el apoyo de las empresas bananeras y gobiernos extranjeros al oponerse a huelgas y mantener al país a una estricta adhesión a los pagos de la deuda. Gobierna con mano dura eliminando la oposición, limitando la libertad de prensa, declarando ilegal el partido comunista y perpetuándose como dictador.

Durante los años treinta empeoró la situación económica nacional ya que hubo una caída de las exportaciones bananeras causada por la recesión mundial, como por brotes de epidemias del banano que afectaron la producción y que generaron desempleo en esa actividad.

Los inmigrantes entre las décadas del 30 y 40 alcanzan las proporciones más altas con respecto a la población total, en los censos de 1926 y 1930 los extranjeros representan el 4.95\% de la población total y en los censos de 1935 y 1940 alcanzan porcentaje cercanos al cuatro por ciento de la población total. Aunque las nacionalidades centroamericanas son mayoría, los europeos y estadounidenses representaron porcentajes importantes del total. 
Los garífunas, grupo étnico negro que llega a la Costa del Caribe de América Central en 1797, después de ser desterrado de San Vicente, ubicándose primeramente en Roatán, Honduras para después desplegarse en las Costas de Belice, Guatemala, Honduras y Nicaragua donde han habitado desde hace más de doscientos años, conservando su cultura, lengua y costumbres. La migración dista de ser una estrategia novedosa en esta cultura, ya que desde fines de la época colonial se sugirió como jornaleros ideales, pero hacia los Estados Unidos de América comienza en 1930. Al inicio los motivos giraban en torno al deseo de suplementar la economía de subsistencia mediante bienes provenientes del exterior, al mismo tiempo que en el país del norte se incrementa la necesidad de mano de obra extranjera en la segunda guerra mundial, ofreciendo empleo en la marina mercante. Al mismo tiempo el deterioro de las condiciones económicas en la década del cincuenta y más recientemente en la década del setenta y posteriores se incrementa la emigración garífuna en la búsqueda de mejores oportunidades de empleo (DED - GTZ, 2004).

La migración garífuna a los Estados Unidos tiene larga data que incluso hizo que la construcción de redes migratorias hayan permitido a sus familiares el poder emigrar legalmente a través del tiempo, por esta razón es difícil encontrar garífunas emigrando ilegalmente, aparte de que la inserción garífuna en el país del norte esta facilitada por parecido físico con los afrodescendientes americanos, como por su bilingüismo (garífuna, español) que incluso facilitaba aprender otra lengua. Una evidencia empírica sobre el peso relativo de las poblaciones afrodescendientes en la emigración hondureña en los Estados Unidos, lo encontramos en el Censo de Población de los Estados Unidos del 2000, que es el que registra los orígenes étnicos de las personas censadas. Honduras registra un total de 14,365 personas que se declararon como afrodescendientes, superior a cualquiera de los otros países del área, con la excepción de Panamá (Baumeister, 2010, p.14).

La economía bananera de Honduras produjo una conexión migratoria importante con los Estados Unidos de América, principalmente con los puertos de la costa este, principalmente New Orleans, incluso los hijos de algunos empleados de las compañías bananeras completaban sus estudios en los Estados Unidos. 


\subsection{Creación del Estado moderno hasta guerra Honduras-EI Salvador.}

Los años cincuenta del siglo pasado asoman potencialidades y deficiencias del país, algunas signos de atraso se manifiestan en una insuficiente infraestructura vial, baja generación de energía eléctrica, niveles bajos de educación, escaso acceso a la salud que es afectada por malaria, infecciones pulmonares e intestinales, una dieta alimenticia limitada, que en general, describe las condiciones de vida de la población hondureña como deplorables (Checchi y Ass, 1959).

Recién salido de la dictadura de Carías en la mitad del siglo pasado se presenció la aceleración de un proceso de modernización, con la asunción de la administración de Juan Manuel Gálvez (1949-1954), ministro de guerra de Carías y abogado de la United Fruit Company. A partir de 1950 se perfilan cambios institucionales, económicos y sociales. Fue creciendo el valor y la importancia del sector público: fundación de la banca estatal; diversificación económica (azúcar, carne, café, algodón, palma africana entre otros); creación del impuesto sobre la renta, otorgamiento de asistencia técnica y crediticia a ganaderos, agricultores; campañas educativas y sanitarias. Toda esta expansión estatal fue posible por los préstamos contraídos con los organismos internacionales de crédito. El sector privado contempla un crecimiento significativo en la manufactura, construcción, comercio y banca. El movimiento cooperativo también se expande. Sin dudas, este período es el nacimiento del Estado moderno hondureño.

Desde 1950 la economía hondureña creció en las siguientes décadas, a excepción de las caídas por la huelga bananera de 1954 y el huracán Fifí en 1974, aunque su mayor crecimiento se experimentó entre 1950 a 1965.

Desde los años 50 y 60, la presión por la tierra se vuelve más aguda en la república de El Salvador hace que Honduras se convierta en tierra atractiva para los salvadoreños. En la década de los años sesenta se percibe que había miles de salvadoreños establecidos en el país. Incluso esta migración particularmente agrícola contribuía con su fuerza de trabajo en la producción bananera, como caficultores y productores de granos básicos, quienes migraban a un país menos desarrollado, pero 
con posibilidades de obtener tierras a bajos costos. Sin embargo, los datos censales de Honduras de 1961 arrojan que de los extranjeros en el país, el 75\% era salvadoreño, alcanzando los 38,002 personas y su tendencia creciente en los censos hondureños venía observándose desde los años treinta del siglo pasado, siendo la primera nacionalidad extranjera en el país en los sesenta, situación que cambió en los censos más recientes.

A raíz de la guerra entre Honduras y El Salvador de julio de 1969 el gobierno expulsa una gran cantidad de salvadoreños a su país. El censo de población de Honduras de 1974 rescata el dato de 100,743 extranjeros que residían en el país, cinco años del censo, o sea en 1969. Desgraciadamente el dato directo de país de nacimiento en 1974 no fue divulgado en ningún documento oficial.

Cuando la inmigración entraba en auge procedente de los países vecinos, también la emigración fue aumentando con el tiempo, y no solo por la emigración garífuna, sino por la colectividad hondureña, que quizás no ha sido estudiado este periodo de emigración, la única evidencia es la que nos muestra los censos de los Estados Unidos de América, que indica que en 1970 esta ocupa el primer lugar del nivel de centroamericanos en el país del norte y en tan solo diez años después ese sitial fue descendido al quinto lugar. Ya en 1980 El Salvador y Guatemala ocupan los primeros lugares, tendencia que mantienen, Honduras alcanza la tercera posición emigratoria regional.

\subsection{Conflictos armados en la región Centroamericana.}

En los años finales de los setenta se recrudece la violencia en la república de Nicaragua por derrocar del poder a Anastasio Somoza, estas tensiones provocan éxodos masivos de nicaragüenses a Honduras. En julio de 1979 es defenestrado del poder, provocando migración en dos direcciones, una de nicaragüenses que regresan a su país y otra que sale de Nicaragua a varios países, entre ellos Honduras.

También a fines de los años setenta e inicios de los ochenta se recrudesen los conflictos internos en El Salvador, provocando una ola inmigratoria hacia varios países entre ellos Honduras, principalmente de refugiados. 
La década de los ochenta en Centroamérica se caracterizó por desplazamientos poblacionales tanto internos como internacionales producto de tensiones polítias que desencadenaron en conflictos armados. Aunque en el país, no se haya presentados los conflictos armados, si prevalecieron movimientos internos en zonas fronterizas, al mismo tiempo que movimientos internacionales procedentes de Nicaragua y El Salvador.

Convertida la nación en receptora de refugiados con por lo menos 37,000 refugiados reconocidos y más de 200,000 desplazados del exterior. Hasta 1988 había 13,325 refugiados salvadoreños en el país, concentrados en campamentos en la zona fronteriza con el Salvador, aunque estimaciones destacan cerca de 20,000 refugiados no reconocidos. La población guatemalteca refugiada ascendía a 430 personas. Los refugiados nicaragüenses en campamentos ascendían a 13,580 en 1988, aunque los refugiados no reconocidos superaban los 180,000 personas (Carranza y Chang, 2002).

E\&A

El censo de población de Honduras de 1988 rescata de los nacidos en el exterior un total de 34,387 personas, de las cuales la mayoría son centroamericanos, principalmente de Nicaragua (44.1\%), El Salvador (22.5\%), Guatemala ((8.9\%). Estos resultados comparados con los censos de 1961 y 1974 donde la población salvadoreña era la de mayor presencia, para 1988 la nicaragüense adquiere supremacía, cambios que no solo están ligados a la búsqueda de seguridad ante los conflictos armados en la región, sino también a la crisis económica generada a raíz de los conflictos.

\subsection{Liberalización de la economía, fenómenos naturales y tendencias recientes.}

Entre 1980 y 1990, como la mayoría de los países de la región, Honduras sufre una crisis generada por la poca diversificación productiva, especialmente porque los principales productos de exportación y fuente de ingresos se concentraban en el banano y el café, aunque se concretaron algunas políticas nacionales para mejorar sus exportaciones como la creación de leyes de zonas libres, regímenes de importación temporal y creación de zonas de procesamiento industrial. 
Cuando en la década del ochenta la demanda internacional y también los precios de compra de los principales productos de exportación disminuyeron, la economía hondureña muestra vulnerabilidad y decae ante la influencia negativa de estos factores externos, por otro lado, el país no contaba con suficiente ahorro interno para sostener su crecimiento económico. Este escenario económico nacional incide en el nivel de vida de la población, generando una baja de los salarios reales y precios más altos para los productos de primera necesidad, lo que provoca inflación, que fue tratado de mitigar a través de medidas económicas asesoradas por organismos internacionales a partir de 1990 con la Ley de Ordenamiento Estructural de la Economía. Así se reconfiguraría la economía nacional, no aumenta significativamente la producción ni la exportación, con relación al crecimiento poblacional, pero genera la disminución del gasto público, cierto grado de diversificación e inversión privada internacional.

La economía hondureña reacciona lentamente a las medidas de ajuste que siguieron el periodo inflacionario. Los rubros de mayor crecimiento en la década fueron los servicios financieros, la construcción y la industria. La agricultura, por su parte, es el principal rubro en relación con su aporte porcentual al PIB (27.5\%), en el año de 1990 representaba el 24.3\%. Los principales productos de exportación siguieron siendo el banano y el café, pero se integra la palma africana y los productos textiles.

Una serie de acontecimientos naturales de gran magnitud contrajeron la economía, el "El Niño" entre 1991 y 1996 en sus zonas costeras. En 1993 hubo una fuerte sequía seguida por una profunda crisis de energía en 1994, relacionada con la producción hidroeléctrica. Ambos acontecimientos provocaron pérdidas cuantiosas para el comercio y el agro. En 1998, el huracán Mitch azota la mayor parte del territorio nacional y seguido hay dos años de sequía en gran parte del territorio.

Desde la década de los noventa las tendencias inmigratorias del reciente pasado por los conflictos en la región, cambian hacia la emigración, incluso los últimos censos de población de 1988 y 2001 muestran volúmenes alrededor de treinta mil personas nacidas en el exterior, que representan porcentajes ínfimos de inmigrantes respecto a la población total, en su mayoría proceden de los países limítrofes, incluso un número no despreciable de estadounidenses (Cuadro No.1). 
Tabla No 1

Honduras: Población nacida en otros países en censos hondureños, 1887-2001

\begin{tabular}{|c|c|c|c|c|c|c|c|c|c|c|}
\hline Nacidos en & 1887 & 1926 & 1930 & 1935 & 1940 & 1945 & 1950 & 1961 & 1988 & 2001 \\
\hline Guatemala & 2,060 & 8,358 & 7,885 & 5,694 & 8,823 & 7,613 & 6,081 & 4,497 & 3,050 & 3,274 \\
\hline El Salvador & 2,000 & 13,452 & 18,522 & 19,168 & 21,309 & 23,029 & 20,285 & 38,002 & 7,733 & 6,291 \\
\hline Nicaragua & 610 & 3,162 & 5,907 & 4,614 & 3,298 & 2,183 & 2,760 & 3,553 & 15,149 & 5,519 \\
\hline Panamá & & & 77 & 95 & 75 & 64 & 105 & 159 & 178 & 283 \\
\hline Costa Rica & 14 & 182 & 178 & 207 & 203 & 162 & 275 & 294 & 557 & 611 \\
\hline Belice & & & 684 & & & & & & 200 & 259 \\
\hline Canadá & & & & & & & 11 & 82 & 149 & 248 \\
\hline $\begin{array}{l}\text { Estados } \\
\text { Unidos }\end{array}$ & 185 & 2,160 & 1,313 & 1,508 & 1,045 & 1,014 & 849 & 1,433 & 2,526 & 4,843 \\
\hline México & 29 & 343 & 424 & 535 & 382 & 300 & 302 & 379 & 746 & 1,114 \\
\hline Caribe & & 177 & 1,111 & 288 & 161 & 118 & 227 & 306 & 294 & 761 \\
\hline Sudamerica & 16 & 22 & 118 & 148 & 218 & 97 & 170 & 393 & 1,578 & 1,991 \\
\hline Europa & 1,252 & 5,544 & 4,358 & 4,710 & 3,664 & 3,204 & 1,217 & 1,598 & 837 & 1,234 \\
\hline Asia & 1 & 1,287 & 1,063 & 1,249 & 1,208 & 1,176 & 382 & 479 & 441 & 1,054 \\
\hline Africa & & & 8 & & & & 39 & & & 25 \\
\hline Otros & & 27 & 632 & 280 & 1,026 & 71 & & 29 & 949 & 469 \\
\hline Total & 6,167 & 34,714 & 42,280 & 38,496 & 41,412 & 39,031 & 32,703 & 51,204 & 34,387 & 27,976 \\
\hline
\end{tabular}

Fuente: Censos de Población de Honduras, 1887-2001, DGECH e INE.

Desde la década de los noventa el flujo de emigrantes hondureños se había venido incrementando por las deplorables condiciones económicas del país, incluso podría decirse que con el fenómeno natural Mitch la emigración internacional adquiere una mayor aceleración y visibilidad, por esta razón no es casualidad que incluso los Estados Unidos de América aprobara el Programa de Protección Temporal (TPS) para frenar la emigración, que se preveía que se acentuaría en la región y principalmente en el país por la destrucción de la infraestructura física y productiva.

En el censo de población hondureño de 2001, se estima que en el 3,34\% de los hogares hondureños alguna persona había salido del país en los tres años anteriores al censo, teniendo como referencia temporal el huracán Mitch, fenómeno natural grabado en la memoria de la población. Los resultados mostraron que los departamentos de Valle, Yoro, Colón, Olancho y Atlántida tenían altas proporciones de hogares con emigrantes, que alcanzaban el 6,28\%. El fenómeno migratorio ya estaba presente en ese año y en algunos municipios, el porcentaje de hogares con emigrantes era entre un $10 \%$ y un 
22\%, principalmente en municipios fronterizos con El Salvador, algunos de Francisco Morazán y de Olancho donde el huracán de 1998 no tuvo efectos devastadores que influyeran directamente en la emigración, lo que indica que ya existía una red de información emigratoria muy fluida que seguramente fue aprovechada por la población (Flores, 2010).

Sobre las causas de la emigración hondureña una serie de factores se conjugan para producir una migración masiva, entre ellos el crecimiento demográfico que no son incorporados en el proceso productivo, un excedente de fuerza de trabajo, salarios inferiores a los demás países de la región, absorción de mujeres en el sector maquilero, excedente laboral en empleo por cuenta propia y familiares no remunerados, niveles de pobreza elevados, llevando a la población a probar con la emigración internacional dar una respuesta a las dificultades de asalariarse en condiciones adecuadas (Baumeister, 2010).

La década del 2000 muestra como el principal producto de exportación del país ya eran "los emigrantes", ya que los flujos de remesas desde el exterior al país se incrementan a través del tiempo, llegando a niveles considerados altos en relación al PIB nacional, que produce que las remesas sean una de las fuentes más importantes de divisas que recibe el país, llegando hasta el $20.4 \%$ en el año 2007. Este incremento en remesas es más palpable entre los años 2005 a 2008 que ha producido ingresos entre los 1,821 a 2,869 millones de dólares. Datos recientes sobre Migración y Remesas 2011 del Banco Mundial ${ }^{1 /}$ reafirman a Honduras en el top mundial de los diez países receptores de remesas del 2009 (utilizando el porcentaje con respecto al PIB) alcanzando el 8 lugar (19\%) y también en la posición 7 de los diez destinos de remesas en América Latina, con 2,700 millones de dólares. Aunque existe una disminución en las remesas en los años 2009 y 2010 los valores giran alrededor de 2,600 millones de dólares.

En esta década se desarrollan dos Módulos de Migración dentro de las Encuestas Permanentes de Hogares y de Propósitos Múltiples de Honduras, EPHPM (2006 y 2010). La última estima 168,786 hogares con emigrantes internacionales, que representan el 9.7\% del total. La población emigrante se estima en 238,669 personas, casi dos tercios son hombres, con destino mayoritario los Estados Unidos (88\%) y otros destinos son España y México. La mayoría es joven, reside en el extranjero desde algún año de la última década y no tienen documentos legales en el exterior (INE, 2011).

1 Datos sobre Migración y Remesas 2011 del Banco Mundial en www. econworldbank.org 
Con los datos del proyecto Investigación de la Migración Internacional en Latinoamérica (IMILA), calculamos que en la década de los setenta habría un número mínimo de 42,737 hondureños residiendo en el exterior, en la ronda de los censos de los ochenta se estimaría unos 50,930 emigrantes. En los años noventa 142,274 hondureños, en la ronda de censos del dos mil 322,696 y en los censos de la ronda del 2010²/ unos 645,532 residiendo en el extranjero (Cuadro No.2).

Tabla No 2

IMILA: Población nacida en Honduras censada en países distintos al nacimiento

\begin{tabular}{|c|c|c|c|}
\hline País y año censal & Nacidos en Honduras & País y año censal & Nacidos en Honduras \\
\hline Argentina (1960) & 88 & México (1970) & 941 \\
\hline Argentina (1970) & $*$ & México (1980) & 1,500 \\
\hline Argentina (1980) & $*$ & México (1990) & 1,997 \\
\hline Argentina (1991) & 138 & México (2000) & 3,570 \\
\hline Argentina (2001) & 142 & México (2010) & 10,991 \\
\hline Belice (1980) & 1,576 & Nicaragua (1971) & 6,919 \\
\hline \multirow[t]{2}{*}{ Belice (1990) } & 2,337 & Nicaragua (1995) & 9,473 \\
\hline & & Nicaragua (2005) & 10,745 \\
\hline Bolivia (1976) & 204 & & \\
\hline Bolivia (1992) & 189 & Panamá (1970) & $*$ \\
\hline \multirow[t]{2}{*}{ Bolivia (2001) } & 195 & Panamá (1980) & 464 \\
\hline & & Panamá (1990) & 623 \\
\hline Brasil (1960) & 80 & Panamá (2000) & 823 \\
\hline Brasil (1970) & 83 & Panamá (2010) & 1,140 \\
\hline Brasil (1980) & 207 & & \\
\hline Brasil (1991) & 300 & Paraguay (1972) & $*$ \\
\hline \multirow[t]{2}{*}{ Brasil (2001) } & 136 & Paraguay (1982) & 27 \\
\hline & & Paraguay (1992) & 35 \\
\hline Colombia (1964) & $*$ & Paraguay (2002) & $*$ \\
\hline Colombia (1985) & * & & \\
\hline \multirow[t]{2}{*}{ Colombia (1993) } & 182 & Perú (1972) & $*$ \\
\hline & & Perú (1981) & $*$ \\
\hline Costa Rica (1963) & * & Perú (1993) & 111 \\
\hline Costa Rica (1973) & 996 & & \\
\hline Costa Rica (1984) & 1,574 & $\begin{array}{l}\text { Rep. Dominicana } \\
\text { (1970) }\end{array}$ & 32 \\
\hline
\end{tabular}

2 Solo incluye Estados Unidos de América, Panamá y México, si tomamos en cuenta los datos del padrón español 2011 habría que sumar 32,418 emigrantes. Aparte hay que agregar otros países. 


\begin{tabular}{|c|c|c|c|}
\hline País y año censal & Nacidos en Honduras & País y año censal & Nacidos en Honduras \\
\hline \multirow[t]{2}{*}{ Costa Rica (2000) } & 2,946 & $\begin{array}{l}\text { Rep. Dominicana } \\
\text { (1981) }\end{array}$ & $*$ \\
\hline & & $\begin{array}{l}\text { Rep. Dominicana } \\
\text { (1993) }\end{array}$ & $*$ \\
\hline Cuba (1970) & * & $\begin{array}{l}\text { Rep. Dominicana } \\
\text { (2002) }\end{array}$ & 143 \\
\hline \multirow[t]{2}{*}{ Cuba (1981) } & $*$ & & \\
\hline & & Uruguay (1975) & $*$ \\
\hline Chile (1970) & 122 & Uruguay (1985) & $*$ \\
\hline Chile (1982) & 179 & Uruguay (1996) & 17 \\
\hline Chile (1992) & 220 & & \\
\hline \multirow[t]{2}{*}{ Chile (2002) } & 244 & Venezuela (1971) & 165 \\
\hline & & Venezuela (1981) & 344 \\
\hline Ecuador (1982) & 104 & Venezuela (1990) & 429 \\
\hline Ecuador (1990) & 117 & Venezuela (2001) & 252 \\
\hline \multirow[t]{2}{*}{ Ecuador (2001) } & 195 & & \\
\hline & & Canadá (1971) & $*$ \\
\hline El Salvador (1971) & 14,290 & Canadá (1981) & 475 \\
\hline El Salvador (1992) & 8,666 & Canadá (1986) & 865 \\
\hline \multirow[t]{2}{*}{ El Salvador (2007) } & 10,387 & Canadá (1996) & 3,935 \\
\hline & & Canadá (2001) & 4,575 \\
\hline Guatemala (1973) & 6,231 & Canadá (2006) & 5,165 \\
\hline Guatemala (1981) & 5,326 & & \\
\hline Guatemala (1994) & 4,634 & $\begin{array}{c}\text { Estados Unidos } \\
(1970)\end{array}$ & 27,978 \\
\hline \multirow[t]{2}{*}{ Guatemala (2002) } & 5,491 & $\begin{array}{l}\text { Estados Unidos } \\
(1980)\end{array}$ & 39,154 \\
\hline & & $\begin{array}{c}\text { Estados Unidos } \\
(1990)\end{array}$ & 108,923 \\
\hline Haití (1971) & 7 & $\begin{array}{c}\text { Estados Unidos } \\
(2000)\end{array}$ & 282,852 \\
\hline Haití (1982) & $*$ & $\begin{array}{l}\text { Estados Unidos } \\
(2010)\end{array}$ & 633,401 \\
\hline
\end{tabular}

$\mathbf{E} \& \mathbf{A}$

Fuente: IMILA y Oficinas de Estadísticas. 
Los Estados Unidos de América es el principal destino emigratorio de los hondureños, tal como lo evidencian todas las fuentes de datos demográficos, en el caso de los censos de población estadounidenses las personas de origen hondureño se ha incrementado en el tiempo, cuando observamos los resultados de la última década el volumen es significativo (Cuadro No.3).

Tabla No 3

Estados Unidos de América: Población de origen hondureño en los censos de población, 1970-2010

\begin{tabular}{|c|c|c|c|c|}
\hline Año & Población & $\begin{array}{c}\text { Incremento } \\
\text { intercensal }\end{array}$ & $\begin{array}{c}\text { \% de incremento } \\
\text { intercensal }\end{array}$ & $\begin{array}{c}\text { Tasa anual de } \\
\text { crecimiento }\end{array}$ \\
\hline 1970 & 27,978 & & & \\
\hline 1980 & 39,154 & 11,176 & 39.95 & 3.3 \\
\hline 1990 & 108,923 & 69,769 & 178.19 & 9.4 \\
\hline 2000 & 282,850 & 173,927 & 159.68 & 8.9 \\
\hline 2010 & 633,401 & 350,551 & 123.94 & 7.7 \\
\hline
\end{tabular}

Fuente: IMILA, CELADE y Census Bureau de EUA, 2010

E\&A

Si comparamos los volúmenes de las personas de origen hondureño con los demás países centroamericanos en los censos de Estados Unidos de América, observamos que en los años setenta los hondureños ocupaban el primer lugar, situación que cambió en los censos posteriores por las emigraciones de salvadoreños, guatemaltecos y nicaragüenses, que tuvieron un componente originado en gran medida, pero no completamente, en los conflictos armados en Centroamérica. Desde los años dos mil las personas de origen hondureño ocupan el tercer lugar en los nacidos centroamericanos en los censos estadounidenses (Cuadro No.4).

Tabla No 4

Estados Unidos de América: Población de origen centroamericano en censos, 1970-2010

\begin{tabular}{|c|c|c|c|c|c|}
\hline País & $\mathbf{1 9 7 0}$ & $\mathbf{1 9 8 0}$ & $\mathbf{1 9 9 0}$ & $\mathbf{2 0 0 0}$ & $\mathbf{2 0 1 0}$ \\
\hline El Salvador & 15,717 & 94,447 & 465,433 & 817,336 & 1648,968 \\
\hline Guatemala & 17,356 & 63,073 & 225,739 & 480,665 & 1044,209 \\
\hline Honduras & 27,978 & 39,154 & 108,923 & 282,852 & 633,401 \\
\hline Nicaragua & 16,125 & 44,166 & 168,659 & 220,335 & 348,202 \\
\hline Costa Rica & 16,691 & 29,639 & 43,530 & 71,870 & 126,418 \\
\hline Panamá & 20,046 & 60,740 & 85,737 & 105,177 & 165,456 \\
\hline Total & 113,913 & 331,219 & 1098,021 & 1978,235 & 3966,654 \\
\hline
\end{tabular}

Fuente: Elaboración propia en base a datos de IMILA, CELADE y Bureau de Census. 
La Encuesta de la Comunidad Americana (ACS), una fuente de datos utilizada en la caracterización de la población estadounidense, también es de suma utilidad para rescatar algunas características de poblaciones de origen foráneo, como principal destino emigratorio hondureño. En el caso de la ACS del 2009 está arroja un total de 625,000 personas de origen hondureño (son inmigrantes hondureños o tienen antepasados de la familia en Honduras). Las personas de origen hondureño son jóvenes, la edad mediana es 28 años, un índice de masculinidad de 118, la estructura por edad muestra dos grupos significativos, los menores de cinco años y la población en edad activa, entre los veinte a cincuenta años (Gráfico No.1).

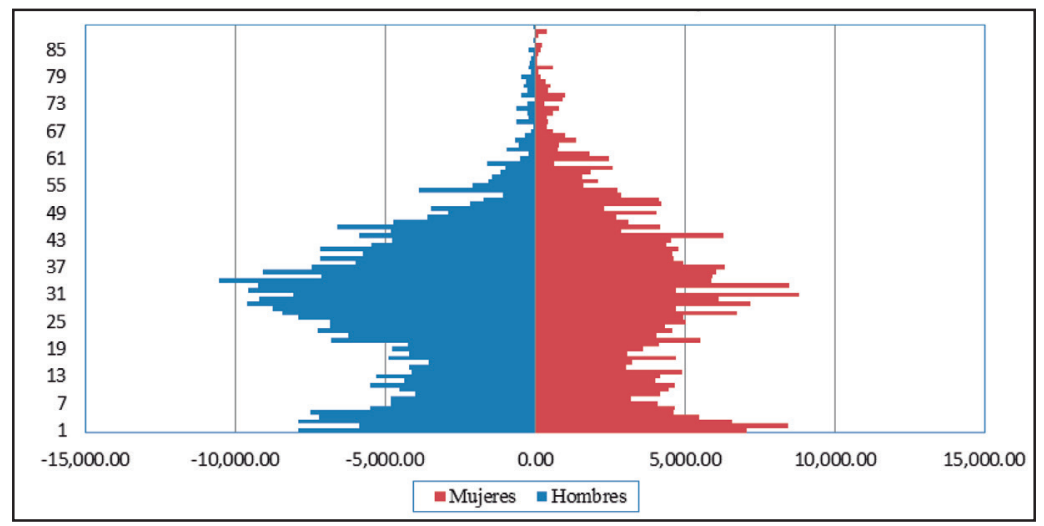

$\mathbf{E} \& \mathbf{A}$

Gráfico 1.

ACS: Pirámide de Población de origen Hondureño en Estados Unidos de América, 2009

Fuente: Elaboración propia en base a datos de la American Community Survey, 2009.

Los hondureños están en octavo lugar en la población de origen hispano en los Estados Unidos. De ellos siete de cada diez nació en el extranjero. Ocho de cada diez llegaron a ese país en 1990 o después, y solamente dos de diez son ciudadanos en el país de destino. Solamente un $40 \%$ de los hondureños hablan inglés con fluidez, el 61\% vive en el sur, principalmente en Florida y Texas, 13\% en California y 9\% en New York. El nivel educativo es más bajo comparado con los promedios de la población hispana, el 49\% de los mayores de 25 años no ha obtenido un diploma de estudios secundarios. 
Las desigualdades de la población de origen hondureño con respecto a la hispana y promedios nacionales se manifiesta en indicadores más deficitarios, tal es el caso de los ingresos anuales que alcanzan los 17,100 dólares, el 27\% de ellos vive en la pobreza, la mitad de ellos no tienen seguro de salud y las tasas de propiedad es de $32 \%$, más agravados que los de la población hispana y de la población de los Estados Unidos de América (PEW Hispanic Center, 2011).

España es un destino extra regional de la migración hondureña que ha adquirido una importancia muy grande en la última década. El padrón español al 1 de enero del 2011 registra un total de 32,418 nacidos en Honduras, de las cuales la mayoría son mujeres, 7 de cada 10 (Cuadro No.5). La población es mayoritariamente joven, ubicada entre los 20 a los 49 años, donde el grupo más representativo es de 25 a 34 años, principalmente de mujeres. Los nacidos en Honduras reside principalmente en la Comunidad de Cataluña, en Barcelona y Girona y en la Comunidad de Madrid.

E\&A

Tabla No 5

España: Población nacida en Honduras por sexo según año, 1998-2011

\begin{tabular}{|c|c|c|c|}
\hline Año & Hombres & Mujeres & Total \\
\hline 1998 & 520 & 1,151 & 1,671 \\
\hline 1999 & 570 & 1,254 & 1,824 \\
\hline 2000 & 694 & 1,542 & 2,236 \\
\hline 2001 & 991 & 2,022 & 3,013 \\
\hline 2002 & 1,355 & 2,593 & 3,948 \\
\hline 2003 & 1,848 & 3,395 & 5,243 \\
\hline 2004 & 2,290 & 4,071 & 6,361 \\
\hline 2005 & 3,001 & 5,321 & 8,322 \\
\hline 2006 & 3,840 & 6,812 & 10,652 \\
\hline 2007 & 5,498 & 10,396 & 15,894 \\
\hline 2008 & 7,844 & 15,829 & 23,673 \\
\hline 2009 & 8,648 & 18,186 & 26,834 \\
\hline 2010 & 8,820 & 20,031 & 28,851 \\
\hline 2011 & 9,392 & 23,026 & 32,418 \\
\hline
\end{tabular}

Fuente: Instituto de Estadística, España. 
La pregunta de emigración utilizada en el censo de población hondureño de 2001, referida a un periodo corto (2001-1998), muestra que los hogares de donde salieron emigrantes ya aparecían un porcentaje no despreciable en los municipios del norte del departamento de Francisco Morazán, como El Porvenir, San Ignacio, Marale, cuyo destino había sido España.

En México la población nacida en otro país asciende a 961,121 personas lo que equivale a $0.85 \%$ de los residentes del país; esta población casi se ha triplicado en los últimos veinte años (Cuadro No.6). Los hombres nacidos en el extranjero son ligeramente mayor que las mujeres (INEGI, 2010, p.20).

Los nacidos en Honduras en los censos de población de México también han tenido un incremento a través del tiempo, principalmente en las dos últimas décadas, incluso pasan de la posición 14 a la 7 del total de extranjeros en el país.

Tabla No 6

México: Población residente nacida en Honduras y otros países, 1895 - 2010

\begin{tabular}{|c|c|c|c|c|c|}
\hline \multirow[t]{2}{*}{ Año } & \multicolumn{3}{|c|}{ Nacidos en Honduras } & \multirow[t]{2}{*}{ Total Extranjeros } & \multirow[t]{2}{*}{$\%$ del Total Extranjeros } \\
\hline & Hombres & Mujeres & Total & & \\
\hline 1895 & 19 & 18 & 37 & 56,355 & 0.07 \\
\hline 1900 & 22 & 3 & 25 & 57,674 & 0.04 \\
\hline 1910 & 78 & 40 & 118 & 117,108 & 0.1 \\
\hline 1921 & 104 & 39 & 143 & 101,312 & 0.14 \\
\hline 1930 & 135 & 84 & 219 & 159,844 & 0.14 \\
\hline 1940 & ND & ND & ND & 67,548 & \\
\hline 1950 & ND & ND & ND & 106,015 & \\
\hline 1960 & ND & ND & ND & 223,468 & \\
\hline 1970 & 508 & 434 & 942 & 192,208 & 0.49 \\
\hline 1980 & 762 & 738 & 1,500 & 268,900 & 0.56 \\
\hline 1990 & 955 & 1,042 & 1,997 & 340,824 & 0.59 \\
\hline 2000 & 1,564 & 2,158 & 3,722 & 492,617 & 0.76 \\
\hline 2010 & & & 10,991 & 961,121 & 1.14 \\
\hline
\end{tabular}

Fuente: Elaboración propia basada en Estadísticas Históricas de México, INEGI. 
El Canadá es otro destino de emigrantes hondureños, mismo que ha aumentado a través el tiempo (Cuadro No.7). De los empadronados en el año 2006, los nacidos en Honduras en su mayoría (69\%) habían llegado a Canadá antes de1996 y en el último quinquenio era reducido los nuevos inmigrantes, que está relacionado con las restricciones de inmigración que cada vez son más exigentes.

Tabla No 7

Canadá: Población de origen hondureño en los censos de población, 1971-2006

\begin{tabular}{|c|c|r|r|r|}
\hline Año & Población & Incremento intercensal & \% de incremento intercensal & Tasa anual de crecimiento \\
\hline 1981 & 475 & & & \\
\hline 1986 & 865 & 390 & 82.11 & 11.6 \\
\hline 1996 & 3,935 & 3,070 & 354.91 & 12.8 \\
\hline 2001 & 4,575 & 640 & 16.26 & 3.0 \\
\hline 2006 & 5,165 & 590 & 12.90 & 2.4 \\
\hline
\end{tabular}

Fuente: IMILA, CELADE, Statistics CANADA.

E\&A

Panamá es un destino de emigrantes hondureños, que aunque no es numeroso, es un destino semicualificado, muestra en el Censo de Población de ese país del 2010 que las 1,140 personas nacidas en honduras, casi las dos terceras partes de ellas tiene entre los 20 a 49 años, más de la mitad es jefe o cónyuge del jefe en su relación de parentesco familiar, el 58\% tiene 6 o más años de educación secundaria, la mitad está ocupado, y de la mitad de ellos trabaja en labores de dirección, profesionales, técnicos y empleados de oficina, la rama de actividad de mayor presencia es el comercio, industria manufacturera, actividades en el hogar como empleadores y construcción, son pocos los inmigrantes desocupados.

\subsection{A manera de conclusión.}

Las tendencias migratorias internacionales de Honduras han tenido comportamientos cambiantes en el tiempo, generados por factores económicos, sociales, culturales e incluso naturales que han llevado a la población hondureña a verse envuelta en el fenómeno migratorio, ya sea como receptora o como agente activo de la migración, tendencia emigratoria que últimamente está en boga. 
Partimos del hecho de como una población que habitaba Honduras al momento del descubrimiento, recibe inmigrantes que además de llevar a cabo la conquista y colonización que duró varios siglos, diezmó la población nativa y con el mestizaje fue conformando la nación, que al mismo tiempo recibió poblaciones negra que vinieron forzadamente a trabajar a las explotaciones en el territorio. Al mismo tiempo, la región Atlántica, fuera del dominio español recibió poblaciones de origen europeo que junto a poblaciones de origen de la islas del Caribe, establece una zona de dominio no español con explotaciones económicas.

Al declararse la independencia de España, aunque se dictan leyes que incitaran a la inmigración, no es sino hasta fines del siglo XIX que con la llamada reforma liberal se atrae inversión extranjera y se crea el enclave minero, consecuentemente se inicia una primera etapa del enclave bananero, que en nuevo siglo culmina en una segunda etapa con la implantación de inversión bananera por las grandes compañías que convierten a Honduras en la república bananera por excelencia. También se producen en estos periodos el ingreso de inmigrantes árabes y judíos que conforman primeramente en los proveedores del comercio nacional y después incursionan en otras ramas de la economía, principalmente en el norte del país, que los convierte en un sector empresarial de poder de decisión. En el sur inmigrantes alemanes incursionan en la economía con éxito, pero son expropiados en la segunda guerra mundial.

Con la crisis económica mundial de los años treinta y sus efectos consecuentes en el país, aprovechando las relaciones comerciales de la costa norte del país y los Estados Unidos empiezan a migrar, caso especial son la población garífuna que inicia su tendencia emigratoria al norte del continente, que años más tarde se vuelve más evidente.

La actividad bananera y después las oportunidades de acceso a tierra a precios accesibles hacen atractiva a la república para los nacionales de El Salvador que inundan el país, y que cuyo desenlace se vuelve más visible con la guerra de las cien horas en 1969. Los conflictos armados en Centroamérica en los países limítrofes producen la llegada de inmigrantes y refugiados que huyen de la violencia, el país se convierte en territorio de llegada de población. Al mismo tiempo, y antes que los países iniciaran su emigración masiva hacia los Estados Unidos de América, ya Honduras llega a ocupar el primer lugar en emigrantes centroamericanos en el país del norte, situación que cambió en el tiempo. 
Con la crisis económica de los años ochenta y con la posterior liberalización de la economía en los años noventa, la emigración se fue haciendo una estrategia de vida de los hondureños, misma que se hace más visible después del paso del huracán Mitch que hace de la migración se acelere y produzca una tendencia migratoria que se mantiene hasta nuestros actuales días.

\section{REFERENCIAS}

Amaya Banegas, Jorge (2000). Los judíos en Honduras. Tegucigalpa: Guaymuras.

Arango, Joaquín (2003). La Explicación Teórica de las Migraciones: Luz y Sombra, Migración y Desarrollo No.1, octubre 2003.

Barahona, Marvin (2005). Honduras en el Siglo XX. Una Síntesis Histórica. Tegucigalpa: Guaymuras.

Baumeister, Eduardo (2010). Bases estructurales de la emigración internacional de los hondureños. Tegucigalpa: RDS.

Carranza, José Venancio y Chang, José (2002). Informes Nacionales de Migración Internacional Honduras. Santiago: CEPAL-OIM-BID.

Corrales, Marvin (2010). Presencia Búlgara en Honduras: Crónica de una investigación histórica, Blog Memoria Gráfica de Honduras. http://fotosantiguashonduras.blogspot.com/2010/03/presenciabulgara-en-honduras.html

Flores Fonseca, Manuel Antonio (1990). La Inmigración en Honduras. Tegucigalpa: UDIP-UNAH.

Flores Fonseca, Manuel Antonio (2010). La Medición Censal de la Migración en Honduras. Notas de Población No. 88. Santiago: CEPAL.

INE (2011). Análisis del módulo de migración en "Comportamiento de la Migración y Remesas Internacionales 2010. Tegucigalpa: Sistema de las Naciones Unidas, INE. 
INEGI (2010). Principales resultados del Censo de Población y Vivienda, Aguascalientes: INEGI.

Murga Frassinetti, Antonio (1978). Enclave y Sociedad en Honduras, Tegucigalpa: UNAH.

Newson, Linda (1992). El Costo de la Conquista. Tegucigalpa: Guaymuras.

DED-GTZ (2004). Remesas y Desarrollo: Lecciones de la Comunidad Transnacional Garifuna. DED-GTZ.

PEW Hispanic Center (2011). Hispanics of Honduran Origin in the United States, 2009. Washington: PEW.

PNUD (2009). Informe sobre Desarrollo Humano 2009, Superando barreras: Movilidad y desarrollo humanos. New York: PNUD.

Welti, Carlos (1997). Demografia I. México: PROLAP-UNAM-CELADE. 